\title{
APLICAÇÃO MULTICÊNTRICA INFORMATIZADA DA COLETA DE DADOS CLÍNICOS NA APENDICITE AGUDA
}

\section{Computerized multicentric application of data collection on acute appendicitis}

\author{
Crhistiano Coleto DRUSZCZ, Emerson Paulo BORSATO, José Simão de Paula PINTO, Osvaldo MALAFAIA
}

ABCDDV/552

Druszcz CC, Borsato EP, Pinto JSP, Malafaia O. Aplicação multicêntrica informatizada da coleta de dados clínicos na apendicite aguda. ABCD Arq Bras Cir Dig 2007;20(3):165-71.

RESUMO - Racional - Um banco de dados clínicos informatizado possibilita melhor forma de coleta, resgate e cruzamento de informações para realização de pesquisa científica. Estudos multicêntricos podem ser gerados a partir da utilização destes mecanismos de forma ágil e prática. $\boldsymbol{O b}$ jetivo - a) Analisar a funcionalidade da base informatizada de dados clínicos das doenças do apêndice ileocecal; b) apresentar os resultados nesta análise para validação do protocolo informatizado das doenças do cólon incorporados no SINPE ${ }^{\mathcal{O}}$ (Sistema Integrado de Protocolos Eletrônicos - INPI $n^{\circ}$ 00051543). Método - Considerando o protocolo específico das doenças do apêndice cecal, coletaram-se informações de prontuários de 862 pacientes com apendicite aguda, de forma multicêntrica, envolvendo três ambientes universitários: Hospital de Clínicas da Universidade Federal do Paraná, Hospital Universitário Evangélico de Curitiba e Hospital do Trabalhador da Universidade Federal do Paraná. O programa de coleta de dados SINPE $^{\circ}$ foi responsável pelo armazenamento, agrupamento e posterior busca das informações. Resultados - O Hospital de Clínicas colaborou com 53,83\% dos pacientes, enquanto que os outros dois com 31,32\% e 14,85\%, respectivamente. A dor abdominal foi o sintoma mais característico e mais presente do quadro clínico dos pacientes que tiveram apendicite aguda confirmada. O hemograma infeccioso (presente em $77,81 \%$ ) e a ecografia, visualizando alteração do apêndice cecal (presente em 67,40\%) foram os exames complementares mais freqüentes para se firmar o diagnóstico referido. A apendicectomia, realizada em $98,43 \%$, foi o procedimento terapêutico padrão para a resolução da doença, tendo sido efetuada por técnica laparotômica em 92,45\% - principalmente pela incisão de MacBurney (74,55\%). As cefalosporinas de segunda geração foram as drogas mais empregadas em associação ao procedimento cirúrgico, seja para profilaxia ou terapêutica das infecções. As complicações pósoperatórias estiveram presentes em 10,32\% dos pacientes, correspondendo principalmente às infecções de parede abdominal (64,28\%). Conclusão - O protocolo informatizado de dados clínicos das doenças do cólon, especificamente em sua parte das doenças do apêndice cecal, é viável e eficaz na manipulação de informações clínicas para produção de estudos científicos uni ou multicêntricos.

DESCRITORES - Informática médica. Coleta de dados. Apendicite.

\section{INTRODUÇÃO}

O desenvolvimento humano torna-se notório quando se observa os avanços que a informática e a tecnologia proporcionam em diversas áreas, como agricultura, administração, engenharia e nas áreas ligadas à saúde da população. Aprimoramentos nos exames diagnósticos e instrumentais cirúrgicos são bons exemplos de como a medicina vem se aproveitando deste avanço tecnológico. Essencial também se faz aplicar às áreas de educação e pesquisa todo este desenvolvimento gerado pela informática.

Acesso ao conhecimento é fundamental. Neste respeito, a fidedignidade dos dados consultados de forma informatizada constitui um pilar para a conseqüente fidedignidade de informações geradas. Trabalhos científicos são usualmente questionáveis, quando usam métodos subjetivos de coleta de informações, métodos

Trabalho realizado no Laboratório de Informática e Multimídia do Programa de Pós-Graduação em Clínica Cirúrgica do Setor de Ciências da Saúde da Universidade Federal do Paraná, Curitiba, PR, Brasil.

Endereço para correspondência: Crhistiano Coleto Druszcz, e-mail: crhistiano_coleto@yahoo.com.br estes geralmente influenciados pelo indivíduo que, por exemplo coleta os dados de um prontuário de paciente. Este fato pode interferir na confiabilidade final de todo o trabalho realizado.

Estudos multicêntricos se destacam pela grande amostragem de dados e também pela diversidade de informações, em comparação a estudos realizados em determinada instituição que podem traduzir informações regionalizadas. São, portanto, mais confiáveis quando comparados a outros métodos de desenvolvimento de pesquisa. Assim, quando bem aplicados, os estudos multicêntricos são significativos e representativos. Adendo único é a necessidade de uma mesma base de dados para a coleta de informações nas diversas instituições envolvidas no estudo.

Visando colaborar com a uniformização dos dados coletados, em 2002, concluiu-se o protocolo informatizado de coleta de dados das doenças do cólon, pelo programa de Pós-Graduação em Clínica Cirúrgica do Setor de Ciências da Saúde da Universidade Federal do Paraná.

Vários estudos já foram realizados referentes às doen- 
ças do apêndice, principalmente apendicite aguda, tendo em vista sua freqüência. É comum deparar-se com estudos científicos bem elaborados e outros com certas falhas, principalmente referentes à metodologia de confecção dos mesmos. Estudos multicêntricos têm sido citados na literatura, porém sem uma base comum na coleta de informações, fato que põe em dúvida a uniformização dos dados adquiridos. Em 1995, Blumeinstein ${ }^{1}$ impelido pela idéia de captação de dados clínicos fidedignos e confiáveis, destacou a necessidade de interligação de sistemas eletrônicos para maior qualidade de informações a serem designadas para a pesquisa.

Uma base de dados computadorizada apresenta-se como melhor alternativa para se coletar dados de forma prospectiva. Várias informações clínicas podem ser armazenadas e selecionadas desta base de dados eletrônica, potencializando a eficácia do trabalho científico prospectivo.

Associando-se protocolos informatizados de pesquisa à realidade de necessidade de normalização de condutas, percebe-se a significativa rapidez e confiança que se pode depositar nas informações geradas por um protocolo eletrônico para desenvolver determinado estudo, com o intuito de responder a tais questionamentos.

Pretende-se, portanto, com o protocolo informatizado de dados clínicos das doenças do cólon, dar contribuição para que a praticidade e eficácia concernente à organização e manipulação dos dados clínicos de pacientes sejam realizadas de forma a prestar informações geradas com idoneidade e veracidade. De certa maneira, cria-se um artifício para que os atuais conflitos ou questões possam ser dirimidos de forma mais ágil, por se possibilitar o acesso às informações de dados clínicos de prontuário de forma mais rápida do que com a realidade atual.

Tendo em mente a grande gama de abordagens realizadas em pacientes com apendicite aguda, aventouse a possibilidade de levantar dados clínicos de pacientes de várias instituições, no intuito de organizar os dados de forma multicêntrica, mas com uma base de coleta de dados comum. Isto possibilitaria substrato de informações para futuros trabalhos científicos confiáveis e de qualidade sobre o assunto.

Os objetivos deste trabalho foram: a) testar a aplicabilidade do protocolo informatizado de dados clínicos das doenças do cólon em caráter multicêntrico, enfocando a apendicite aguda; b) demonstrar e analisar os resultados coletados em três hospitais universitários; c) validar o protocolo informatizado de dados clínicos das doenças do cólon, após aplicação e análise das informações obtidas sobre apendicite aguda.

\section{MÉTODOS}

Três fases distintas estiveram envolvidas na confecção desta pesquisa.

\section{Primeira fase: Escolha dos três centros hospitalares para coleta de dados clínicos}

Ela foi baseada em alguns critérios comuns: facilidade na coleta dos dados de forma retrospectiva, facilidade no levantamento de informações dos prontuários médicos de pacientes internados, permissão e colaboração na aplicação do protocolo eletrônico das doenças do cólon.

Foram três os centros médico-hospitalares escolhidos: Hospital de Clínicas da Universidade Federal do Paraná (HCUFPR), Hospital Universitário Evangélico de Curitiba (HUEC) e Hospital do Trabalhador da Universidade Federal do Paraná (HTUFPR).

\section{Segunda fase: Levantamento e coleta dos dados utilizando o protocolo informatizado das doenças do apêndice cecal}

Tendo sido escolhidos os centros universitários, foi instalado neles o protocolo informatizado de dados clínicos das doenças do cólon para utilização do protocolo específico das doenças do apêndice cecal.

A instalação do programa "SINPE" (Sistema Integrado de Protocolos Eletrônicos - Registro INPI: 0051543 propriedade intelectual de um dos autores - Malafaia,O) foi efetivada em $2004^{12}$. Teve uma base de receptação e funcionamento comum que se situou no Laboratório de Informática e Multimídia do Programa de Pós-Graduação em Clínica Cirúrgica do Setor de Ciências da Saúde da Universidade Federal do Paraná em Curitiba,PR.

A linguagem padronizada de programação foi $\mathrm{CH}$.

Como particularidade desta base eletrônica de dados, ou seja, o protocolo informatizado de dados clínicos das doenças do cólon ${ }^{5}$, a sua criação se deu a partir da reunião de informações referentes às doenças do cólon na literatura em um depósito comum que se denominou protocolo mestre. A partir deste, as informações foram organizadas e agrupadas de forma minuciosa em protocolos denominados específicos, já que continham peculiaridades de determinadas doenças ou grupo de doenças. No total, foram criados 16 protocolos específicos das doenças do cólon.

No presente estudo, enfocou-se apenas um dos específicos, o das doenças do apêndice cecal, que serviria como exemplo de como todas as outras subunidades funcionariam.

De forma lógica, desenvolveu-se um sistema para proteção dos dados a serem manipulados no protocolo referido. Este sistema consistiu no provimento de um login para cada usuário e este determinaria a sua própria senha para entrada no software.

Dois níveis de autenticação ao sistema são evidenciados: um que verifica a classificação do usuário (comum ou super-usuário) e outro que verifica o tipo de permissão liberada ao usuário referente a cada protocolo, individualmente. O usuário classificado como comum, necessita da autorização previamente concedida por um super-usuário, que, por sua vez, possui acesso irrestrito às informações e operacionalidade do software. Quatro são os tipos de permissão que podem ser direcionadas pelo super-usuário9: a) administrador: possui acesso irrestrito, pode acessar os protocolos mestre e específico, coletar informações e realizar pesquisa de dados clínicos; b) visualizador: visualiza o protocolo informatizado como um todo, porém, não tem 
permissão para alterar os dados implícitos ao protocolo ou alterar os dados referentes à coleta de informações de prontuário; c) coletor: tem apenas permissão para acessar o item de coleta de dados; d) pesquisador: tem permissão de acesso ao item "pesquisa", podendo apenas coletar dados clínicos com objetivo científico.

Apresentam-se, na seqüência, figuras referentes ao protocolo informatizado de dados clínicos específico das doenças do apêndice cecal. A Figura 1 exibe os sete itens principais a serem abertos e preenchidos pelo usuário para a coleta de informações clínicas dos pacientes. Todos os dados clínicos são coletados por se clicar sobre o item relevante.

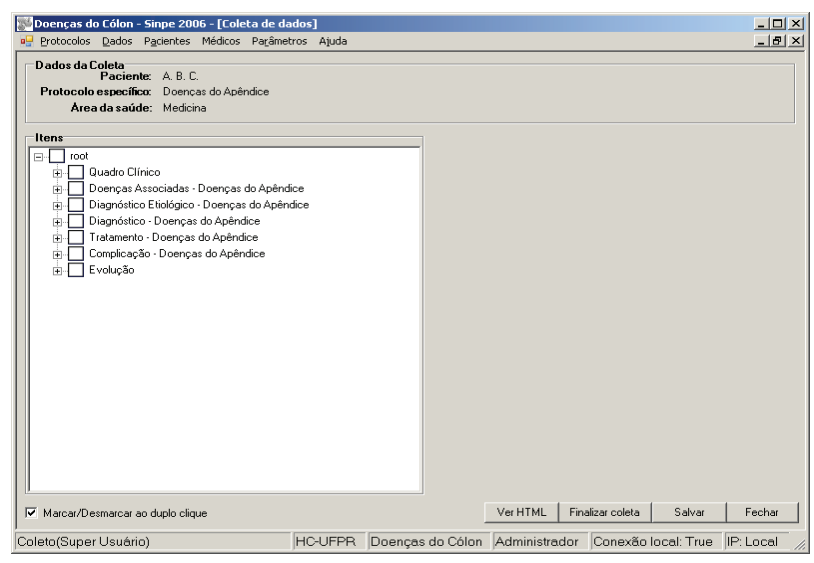

FIGURA 1 - Protocolo específico das doenças do apêndice com os itens gerais relacionados. Estes itens devem ser abertos para a devida coleta dos dados clínicos do paciente

A Figura 2 revela o preenchimento das complicações observadas na evolução de um determinado paciente. Refere-se a um exemplo da forma a serem armazenados os itens relevantes da coleta de informações dos pacientes cadastrados. Observa-se também, a forma a serem pesquisadas e cruzadas as informações para utilização na confecção de um trabalho científico, isto é, os itens de seleção de pesquisa e as respectivas teclas a serem acionadas.

\section{Terceira fase - Análise das informações coletadas e respectivos resultados}

Ela se fez de forma retrospectiva (meados de 2004 a meados de 2006), referindo-se aos prontuários de pacientes submetidos à apendicectomia por apendicite aguda em seus variados estágios de evolução, nos três centros universitários cadastrados no programa.

$\mathrm{Na}$ prática, os resultados repassam os percentuais acionados de cada item durante todo o processo de coleta de dados clínicos dos prontuários dos pacientes. Exemplificando: quantidade específica de vezes que um determinado sintoma, ou exame diagnóstico ou complicação cirúrgica esteve presente na amostra total de pacientes.

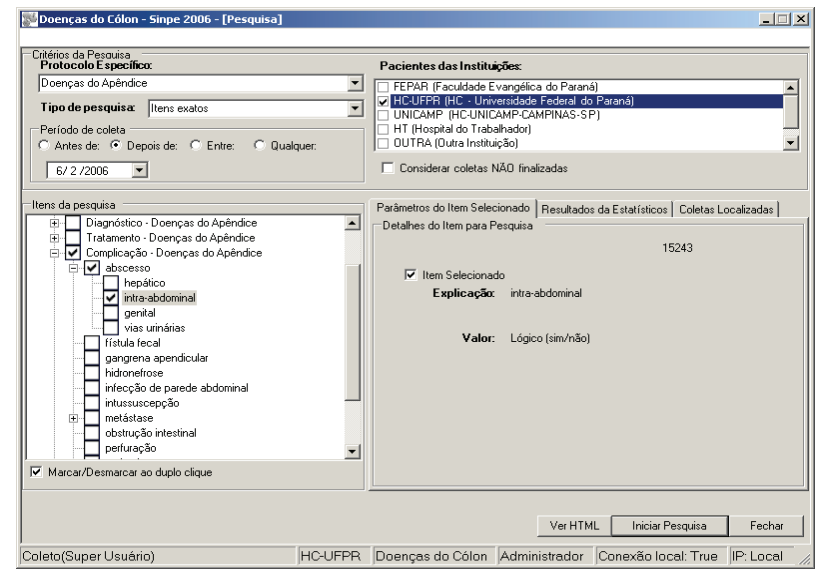

FIGURA 2 - Seleção das informações pelo "clique" sobre o item a ser pesquisado. Este é o modo utilizado para o levantamento dos dados coletados no protocolo informatizado, definido o período de coleta e itens de interesse

Disponibiliza-se o cruzamento de dados como opção gerada pelo programa para o usuário pesquisador, para tentar relacionar algumas informações. Por exemplo, podese cruzar um determinado sinal/sintoma, como "febre", com o aparecimento de uma determinada complicação pós-operatória, como "infecção de ferida cirúrgica".

Realizou-se o agrupamento das informações adquiridas nas três instituições envolvidas no estudo visando melhor análise da amostra dos dados coletados. Partindo deste ponto, concretizou-se o levantamento dos dados apresentados nos resultados, de maneira imediata, com a utilização do próprio programa em seu módulo de pesquisa.

\section{RESULTADOS}

Foram avaliados 862 pacientes submetidos à apendicectomia por apendicite aguda. Especificamente, 464 pacientes foram provenientes do HCUFPR, 270 do HUEC e 128 HTUFPR. Assim, O HCUFPR colaborou com 53,83\% dos pacientes, enquanto que o HUEC e HTUFPR contribuíram com $31,32 \%$ e $14,85 \%$, respectivamente.

O Gráfico 1 sintetiza as principais queixas observadas no atendimento inicial de cada paciente.
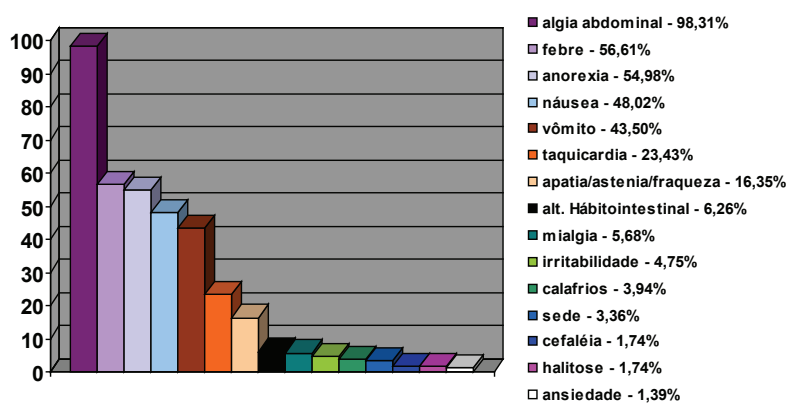

GRÁFICO 1 - Principais sintomas do quadro clínico observado nos pacientes com apendicite aguda 
O Gráfico 2 apresenta as principais alterações visualizadas nos exames laboratoriais dos pacientes envolvidos no presente estudo.

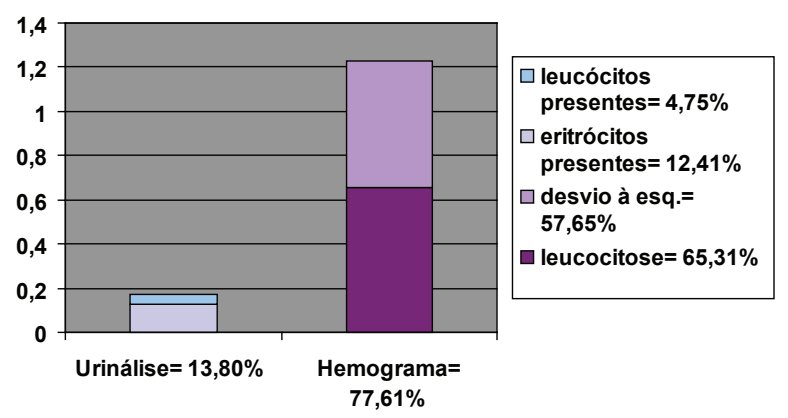

GRÁFICO 2 - Alterações mais relevantes nos exames laboratoriais.

O Gráfico 3 descreve, de forma decrescente em freqüência, as alterações mais percebidas para o diagnóstico da apendicite aguda na ecografia abdominal.
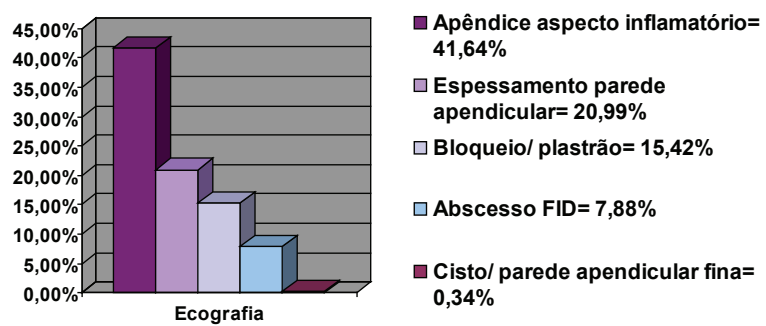

GRÁFICO 3 - Alterações verificadas na ecografia abdominal nos três hospitais universitários envolvidos no estudo

A radiologia, seja efetuada de forma simples ou com a utilização de contraste, corrobora para validação diagnóstica da apendicite aguda. O Gráfico 4 revela os principais achados e as suas respectivas freqüências de manifestação nos exames radiológicos explicitados.

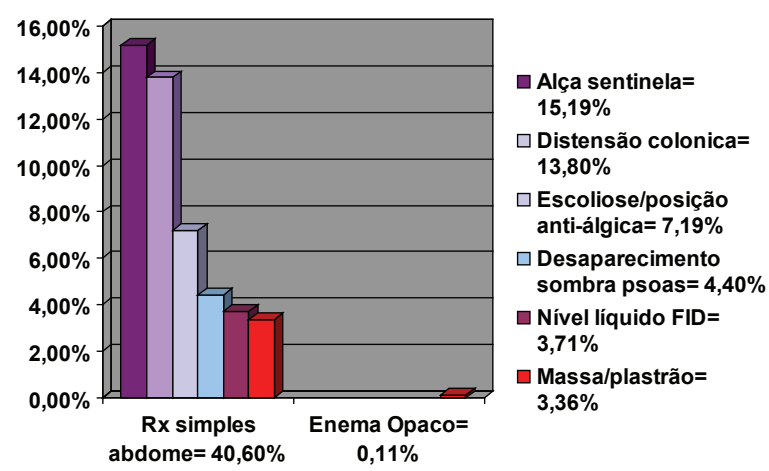

GRÁFICO 4 - Alterações manifestadas nos exames radiológicos
Os Gráficos 5 e 6 retratam os aspectos cirúrgicos do item "Tratamento", no protocolo específico das doenças do apêndice. O primeiro, enfatiza as principais vias de acesso para abordagem do apêndice cecal e suas freqüências de apresentação no estudo. Já o segundo, salienta os principais procedimentos operatórios utilizados para resolução da doença.

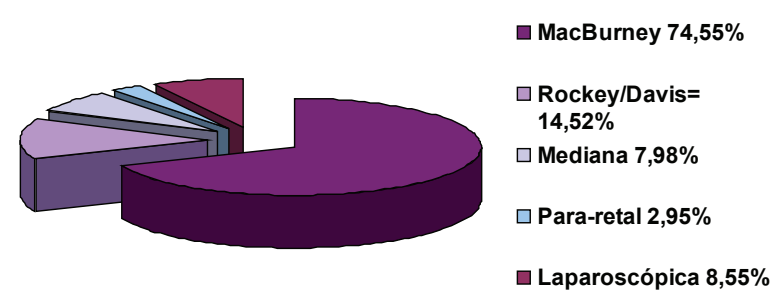

GRÁFICO 5 - Principais vias de acesso cirúrgico utilizadas para acesso ao apêndice cecal no tratamento da apendicite aguda
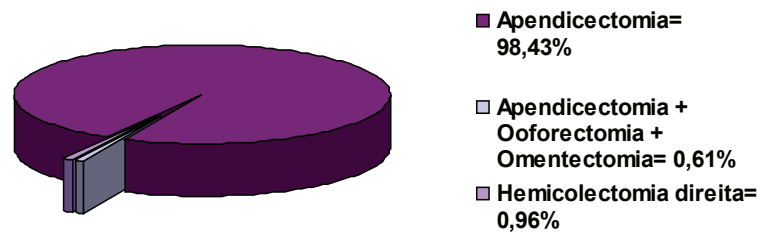

GRÁFICO 6 - Abordagem cirúrgica envolvida na resolução da apendicite aguda

O Gráfico 7 demonstra a antibioticoterapia ou antibioticoprofilaxia. Evidencia-se a percentagem de adoção das classes de antibiótico na manipulação dos 862 pacientes envolvidos, seja no intuito de auxiliar o tratamento ou prevenir complicações.
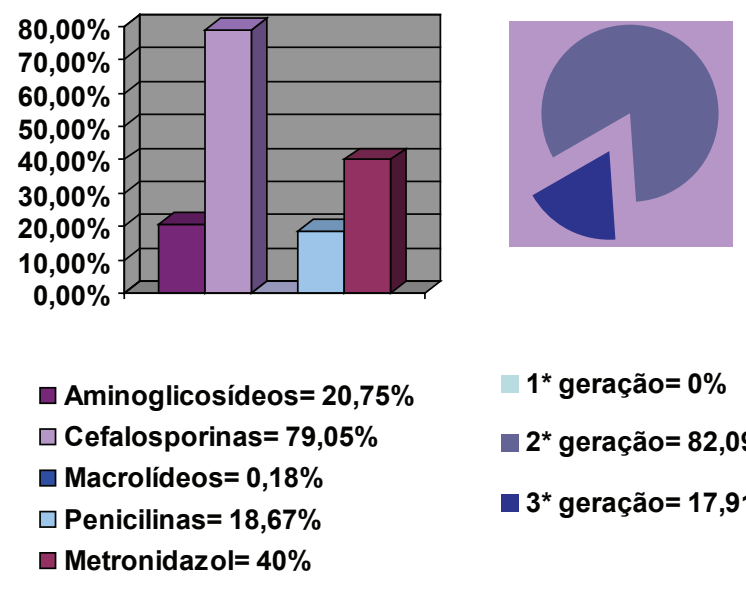

GRÁFICO 7 - Classes de medicação antibiótica utilizadas para antibioticoterapia ou antibioticoprofilaxia 
Finalmente, o Gráfico 8 enfoca o período pós-operatório e de acompanhamento da evolução dos pacientes envolvidos no ensaio científico, fazendo um adendo no que se refere ao quesito "Complicações". Assim, se esclarece quais as complicações pós-operatórias estiveram mais associadas ao período de convalescença dos 862 pacientes.
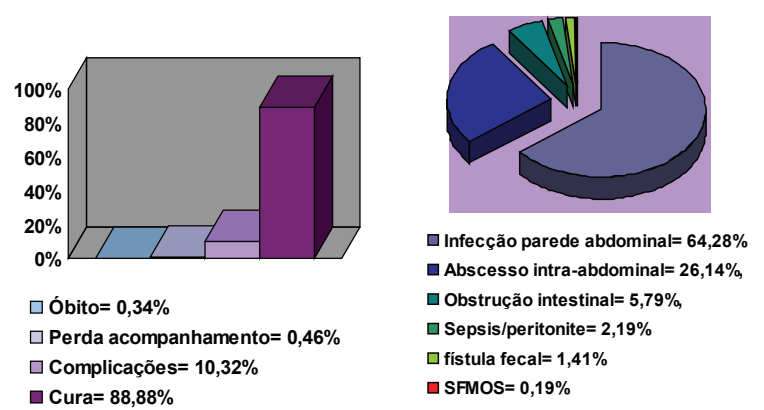

GRÁFICO 8 - Apresentação da evolução pós-operatória dos pacientes submetidos à apendicectomia e complicações mais prevalentes no período referido

\section{DISCUSSÃO}

A tecnologia vem a cada dia surpreendendo os humanos com novidades das mais variadas. $\mathrm{Na}$ área da saúde, o desenvolvimento de aparatos protéticos e eletrônicos vem aprimorando o desenvolvimento clínico-cirúrgico e facilitando o estilo de vida do homem. Em defesa disso, a pesquisa surge como um dos fatores primordiais, pois torna possível a correlação de métodos inovadores com seus respectivos resultados e permite realizar suporte consistente de mecanismos definitivamente eficazes. Torna-se, portanto, imprescindível manterem-se métodos de avaliação e pesquisa cada dia mais desenvolvidos. A informática trouxe consigo possibilidades múltiplas de associar a medicina à modernização eletrônica globalizada. Desta maneira, quando se abordam formas de coletar dados clínicos, uma base de dados eletrônica parece surgir como forma competente de oferecer segurança e agilidade associado à credibilidade na confecção dos estudos científicos ${ }^{6,10,14}$.

Apesar de oferecerem mais vantagens no ponto de vista qualitativo, os trabalhos científicos baseados em programas informatizados são dispendiosos, exigindo considerável quantidade de dinheiro para manter todo o aparato necessário para o seu bom funcionamento: estações de computação ou microcomputador, programas atualizados e equipe de pessoal que seja especializado com informática médica, possuindo conhecimento e experiência sobre o assunto. A necessidade de treinamento de pessoal referente às coletas dos dados clínicos é outro fator limitador, pois exige tempo para prover conhecimento sobre o programa e habilitação para a sua aplicação. Fica claro, desta maneira, porque ainda existe certa limitação na utilização de bases informatizadas de dados clínicos ao longo do mundo todo.

Os grandes bancos de dados de pesquisa clínica apa- rentam ocupar nichos específicos e valiosos no espectro de pesquisa médica. Alguns autores norte-americanos definem os protocolos de pesquisa e os bancos de dados como fontes de alta qualidade para o desenvolvimento da pesquisa científica ${ }^{8,11}$. Porém, não existe lógica em se possuir um grande banco sem a possibilidade de utilizá-lo devidamente, já que é impossível se manejar algo que não se pode medir ${ }^{13,15}$.

Alerta deve ser dado pelo autores deste trabalho neste momento: "O desenvolvimento de um protocolo informatizado para pesquisas clínicas não torna obsoleto $\mathrm{o}$ preenchimento detalhado do prontuário médico do paciente!". Este alerta é pertinente porque um protocolo informatizado tem por objetivo dinamizar a realização de pesquisa científica provendo forma de se ganhar tempo e eficácia sem o detrimento da confiança e segurança de determinado estudo. Não substitui também, o prontuário médico, que é documento legal e individualizado de determinado paciente em relação às afecções que o acometeram.

Credibilidade pode acompanhar qualquer centro que se torna referência por seus estudos. A informática pode possibilitar esta transformação quando aprimora a metodologia de confecção dos estudos científicos. Bom exemplo pode ser observado na cidade de Birmingham, onde a Universidade do Alabama tornou-se centro de referência em estudos relacionados aos pacientes portadores do vírus HIV. Isto ocorreu após a manutenção de um programa de protocolos informatizados que facilitou a coleta de dados clínicos destes pacientes e possibilitou a publicação de uma série de estudos bem fundamentados no assunto ${ }^{3,7}$.

O protocolo informatizado pode ser implantado em um único centro para referendar os dados deste. Porém, abre-se grande oportunidade de qualificação de um estudo científico se ele é implementado em vários centros. É bem verdade que pode ser um desafio, se diferentes centros possuírem diferentes sistemáticas e rotinas de trabalho. No entanto, se o objetivo for o de criação de um estudo científico mais consistente, certamente a opção de multicentricidade se torna bem interessante, podendo desviar certas tendências ou resultados pertinentes à rotina de um determinado serviço ou centro hospitalar.

Este protocolo informatizado de pesquisa foi criado com o intuito de abolir a coleta de dados que permitam diversas interpretações, possibilitando ainda, facilidade e agilidade no processo de coleta. Desta forma, o programa foi criado utilizando-se uma base de dados com informações fechadas, ou seja, basta ao usuário que for coletar os dados, clicar sobre a lacuna correspondente à informação vigente, num determinado caso clínico, para se efetuar a coleta e armazenamento da informação no protocolo. Esta característica faz com que informações subjetivas (de caráter descritivo) não sejam armazenadas, dirimindo futuras interpretações dúbias por parte dos pesquisadores para determinada coleta. Este cuidado é necessário para se alcançar resultados satisfatórios. A simplicidade no esquema de coleta de dados clínicos dinamiza o processo, tendo em vista o fácil entendimento do procedimento a ser realizado pelos usuários coletores. 
Faz-se necessário comentar que o auxílio técnico é essencial, não somente na escolha das ferramentas a serem utilizadas na confecção do protocolo, mas também na manutenção e adequação do programa às novidades que, inevitavelmente, surgem de forma rápida no campo da computação.

Segurança absoluta é algo que pode ser considerado utópico já que vários modelos de agressão a sistemas informatizados são criados a cada instante por indivíduos mal intencionados, principalmente quando associados à Internet ${ }^{2,4}$. No entanto, com o objetivo de evitar ao máximo a violação e proporcionar grau adequado de segurança, o "software" utiliza mecanismos de proteção ao acesso (senha e login), referenda-se na utilização da linguagem $\mathrm{C} \#^{\mathcal{O}}$ e possui um banco único de dados, além de outros meios, para tornar satisfatória a segurança da coleta de dados via Internet.

Em análise geral, observa-se que os dados obtidos desta amostra de 862 pacientes com apendicite aguda, confirmam os procedimentos padronizados mundialmente quanto ao diagnóstico, tratamento e acompanhamento pós-operatório encontradas na literatura mundial.

A concretização de uma base de dados confiável é essencial para o desenvolvimento de trabalhos científicos e temas de pesquisa. Desta maneira, nada mais apropriado do que a utilização de um sistema informatizado para se criar tal base de dados. Com o emprego deste recurso de maneira prospectiva, a confiabilidade dos dados torna-se ainda maior, por excluir a possibilidade de erros ou vícios na coleta das informações dos prontuários dos pacientes.

A forma ideal para o desenvolvimento das pesquisas clínicas é com o emprego das informações que foram sendo acumuladas no decorrer do tempo, através da coletas elaboradas e minuciosas concomitante ao internamento dos pacientes. Peculiaridades concernentes às informações não passam despercebidas ou deixam de ser coletadas, tendo em vista a proximidade do internamento.

Infelizmente por questões técnicas, a exemplificação da aplicabilidade do protocolo informatizado das doenças do cólon, com a coleta de pacientes com apendicite aguda, ou seja, utilizando-se apenas um protocolo específico de todo o sistema, se formalizou de forma retrospectiva. Assim, a chance de vícios na coleta dos resultados pode ser considerada maior. A explicação para o ocorrido se dá pelo escasso período de tempo associado às necessidades de aprimoramento no conteúdo técnico de informática do "software".

Não obstante este fato, a fonte de informações organizada e detalhada constantes nesta base de dados informatizada concretiza fonte muito grande de conteúdo para a elaboração de trabalhos científicos, podendo-se dispor das mais vastas variantes de cruzamento de dados.

Uma fonte com tal qualidade e versatilidade torna-se imprescindível para o aprimoramento e a qualificação cada vez maior das pesquisas realizadas pelos centros interessados em pesquisa científica. A presença de um protocolo informatizado nestes centros certamente sedimenta a qualificação dos mesmos no ambiente científico.

$\mathrm{O}$ pesquisador se depara constantemente perante um questionamento: "É a minha linha de raciocínio lógica?" Desta forma, muitas vezes ele se assemelha a um inventor que testa suas criações para averiguar se funcionam efetivamente ou se não passam de meros devaneios em busca de um ideal.

E foi exatamente o que se procurou fazer com o protocolo informatizado das doenças do cólon. Quando da sua criação, apresentou-se como forma de aprimorar, agilizar e qualificar os trabalhos científicos que dele fossem elaborados. A idéia parecia interessante, mas a real aplicabilidade do sistema informatizado ainda era um desafio a ser transposto. Dessa forma, o presente estudo voltou-se para a comprovação da eficácia do "software" criado. E, definitivamente, ele demonstrou satisfazer todas as necessidades a que um pesquisador está submetido no transcurso de elaboração de um trabalho de pesquisa científica.

Assim, espera-se que mais pesquisadores possam se aproveitar deste meio eficiente de organizar e criar pesquisas clínicas utilizando-se de um método informatizado de provimento de informações.

\section{CONCLUSÕES}

a) O protocolo informatizado é aplicável multicentricamente para a apendicite aguda; b) a avaliação dos resultados e a obtenção de informações de importância para o estudo se tornaram factíveis; c) foi possível realizar a validação do protocolo das doenças do apêndice. 
Druszcz CC, Borsato EP, Pinto JSP, Malafaia O. Computerized multicentric application of data collection on acute appendicitis. ABCD Arq Bras Cir Dig 2007;20(3):165-71.

ABSTRACT - Background - A clinical database allows a better way to collect and, consequently, search and cross information to scientific researches. Multicentric studies can be easily created by using this mechanism. Aim - a) To analyze the functionality of the computerized database of the appendix diseases; $b$ ) to show the results of the analyzed data to validate the computerized database of the colon diseases incorporated to SINPE $\mathbb{R}$ (Computerized Database Integrated System - INPI 00051543). Method - Information provided by 862 patients with acute appendicitis has been collected into the specific protocol of the appendiceal diseases, from three university centers: Hospital de Clínicas of the Universidade Federal do Paraná, Hospital Universitário Evangélico de Curitiba and Hospital do Trabalhador of the Universidade Federal do Paraná. SINPE@ was the software used to store, to select and to search information thereby compared to general literature. Results - The Hospital de Clínicas supported $53,83 \%$ of the patients, meanwhile, the other two included $31,32 \%$ e $14,85 \%$, respectively. Abdominal pain was the most common and characteristic symptom in patients who had confirmation of acute appendicitis. Infectious hemogram (in 77, 81\%) and abdominal ultrasound showing parietal abnormalities of the appendix (in 67, 40\%) were the diagnostic exams more frequently adopted to prove the referred diagnosis. Appendectomy, applied in $98,43 \%$ of the patients, was the standard therapeutic proceeding. The MacBurney incision, applied in $74,55 \%$ of all conventional surgeries, was the most frequent form to make access to the peritoneal cavity. Second generation cephalosporin was the usual drug associated to surgery, even for prophylaxis or therapeutics, on the infectious process. $10,32 \%$ of the patients developed a kind of post-operative complication, being parietal infection $(64,28 \%)$ the most prevalent. Conclusions- The clinical database's computerized protocol of colon diseases, specifically on its appendiceal diseases' protocol, was considered efficient and viable to manipulate clinical information objecting simple or multicentric scientific researches production.

HEADINGS - Medical informatics. Data collection. Appendicitis.

\section{REFERÊNCIAS}

1. Blumenstein BA. Medical research data. Control Clin Trials. 1995;16:45355.

2. Dick RS, Steen EB. Essential technologies for computer-based patient records: a summary. Berlin; Springer-Verlag, 1992. p.229.

3. Dickersin K. How important is publication bias? A synthesis of available data. AIDS Educ Prev. 1997;9 (Supl 1)15-21.

4. Dickersin K. The existence of publication bias and risk factors for its occurrence. JAMA. 1990;263:1385-9.

5. Druszcz CC. Base eletrônica de dados clínicos das doenças do cólon [dissertação]. Curitiba: Universidade Federal do Paraná; 2002.

6. Kohane MG. Clinical databases and critical care research. Critical Care Clin. 1996;10:37-51.

7. Lee JY. Uses of clinical databases. Am J Med Sci. 1994;308:58-62.

8. Lubin JH. Randon exposed underground miners and inverse dose-rate (protraction enhancement) effects. Health Physicist. 1995;14:2057-79.

9. Malafaia O, Borsato EP, Pinto JSP. Manual do usuário do SINPE®. Curitiba: Universidade Federal do Paraná; 2003.
10. McDonald C J, Tierney WN, Overhage M. The Regentrief Medical Record System: 20 years of experience in hospitals, clinics and neighborhood health centers. Medical Data Computing 1992;9:206-17.

11. Nakamura RM. Technology that will initiate future revolutionary changes in health care and clinical laboratory. J Clin Lab Anal. 1999;13:49-52.

12. Pinto JSP. Interface de visibilização de informações para o Sistema Integrado de Protocolos Eletrônicos (SINPE®). Curitiba: Universidade Federal do Paraná; 2005.

13. Rocha-Neto JM, Rocha-Filho JM. Serviço de arquivo médico e estatístico (computadorizado) como meio de aprimoramento de ensino, da pesquisa e da administração. Revista AMRIGS. 1983;27:492-4.

14. Sitting DF. Grand challenges in medical informatics? J Am Med Infor Assoc. 1994;1:412-3.

15. Stewart LA. On behalf of the Cochran Working Group on Meta-Analyses Using Individual Patient Data. Practical methodology of meta-analyses (overviews) using updated individual patient data. Statement Med. 1995;14:2057-79.

Conflito de interesse: não há Fonte financiadora: não há Recebido para publicação em: 03/04/2007 Aceito para publicação em: 30/05/2007 